\title{
Interpretações de Significado em Educação Matemática
}

\author{
Interpretations of Meaning in Mathematical Education
}

Ole Skovsmose

ORCID iD 0000-0002-1528-796X

\begin{abstract}
Resumo
Como ponto de partida para uma interpretação de significado em Educação Matemática, consideremos duas crianças brasileiras fictícias, Paulo e Eloísa. Elas têm experiências de vida diferentes, e tais diferenças referem-se às condições sociais, econômicas, culturais e religiosas, e também às visões, suposições e preconceitos que poderiam construir algo como significativo ou sem sentido. A fim de articular a formação sociopolítica das experiências de significado, apresento uma interpretação do significado com referências no foregrounds dos estudantes. A ideia básica é reconhecer que os foregrounds são formados por uma série de fatores sócio-políticos. Esses fatores são discutidos em termos de foregrounds polarizados, destruídos, amputados, direcionados e multiplicados.
\end{abstract}

Palavras-chave: Foregrounds Polarizados. Foregrounds Destruídos. Foregrounds Amputados. Foregrounds Direcionados. Foregrounds Multiplicados.

\begin{abstract}
As a starting point for an interpretation of meaning in mathematics education, let us consider two fictitious Brazilian children, Paul and Eloisa. They differ in life experiences, and such differences refer to social, economic, cultural, and religious conditions, and also point of views, assumptions and prejudices that could construct something meaningful or meaningless. In order to articulate the socio-political formation of meaning experiences, I present an interpretation of meaning with references in the students' foregrounds. The basic idea is to recognize that foregrounds are formed by a number of socio-political factors. These factors are discussed in terms of polarized, destroyed, amputated, directed, and multiplied foregrounds.
\end{abstract}

Keywords: Polarized Foregrounds. Destroyed Foregrounds. Amputee Foregrounds. Directed Foregrounds. Multiplied Foregrounds.

\section{Introdução}

Geralmente, acorda-se que é importante organizar a Educação Matemática de forma que ela seja significativa para os estudantes. Concordo com isso, no entanto não existe um acordo sobre como isso pode se configurar na prática. Isso não surpreende, já que as

\footnotetext{
* Doutor em Educação Matemática, Royal Danish School of Educational Studies, Copenhague, Dinamarca. Professor Emérito, Universidade de Aalborg, Dinamarca. Professor Colaborador, Universidade Estadual Paulista "Júlio de Mesquita Filho" (Unesp), Rio Claro, São Paulo, Brasil. Endereço para correspondência: Rua 4, 555, apartamento 44, Rio Claro, São Paulo, Brasil, CEP: 13500-030. E-mail: osk@learning.aau.dk.
} 
divergências quanto às interpretações da própria noção de significado são profundas. ${ }^{1}$

A discussão sobre significado tem raízes filosóficas profundas. Uma teoria da filosofia diz que o significado de um conceito deve ser especificado através de suas referências. Essa teoria referencial do significado foi formulada por Platão e pelo platonismo em geral. Para identificar o significado de um conceito, o que vale também para um conceito matemático, é preciso identificar referências adequadas no mundo das ideias. Uma elaboração particular de tal teoria referencial foi fornecida por Gottlob Frege, que afirmou que os conjuntos são as referências apropriadas para conceitos matemáticos.

Uma interpretação radicalmente diferente de significado foi apresentada por Ludwig Wittgenstein $(1922,1997)$. Enquanto em Tractatus, ele elaborou uma teoria referencial do significado, nas Philosophical Investigations, ele sugeriu que o significado de um conceito precisava ser esclarecido em termos de seu uso.

Essas duas interpretações de significado foram cuidadosamente elaboradas na Educação Matemática. Durante a década de 1960, uma teoria referencial inspirada por Frege acompanhou a implementação da chamada Matemática Moderna: o significado dos conceitos matemáticos seria esclarecido em termos da teoria dos conjuntos. Assim, o significado de, digamos, função, deveria ser estabelecido por meio de um caminho que introduzisse as noções de conjunto, pares ordenados, antes de chegar na própria noção de função. Depois que o movimento da Matemática Moderna se desvaneceu, várias outras tentativas foram feitas para estabelecer o significado através das referências de conceitos. O pressuposto geral é que o significado de um conceito complexo pode ser adequadamente compreendido em termos das referências de seus elementos constitutivos. ${ }^{2}$

Já a teoria apresentada por Wittgenstein assume que os significados de conceitos de Matemática são estabelecidos, em primeiro lugar, através do uso de tais conceitos. A ideia geral é que os estudantes experienciam significado quando trabalham com as aplicações da Matemática e fazem relação, por exemplo, com fazer compras, planejar viagens, calcular impostos, etc. Uma elaboração mais aprofundada da teoria do significado, em termos de uso, encontra-se nas muitas propostas de Modelagem Matemática. ${ }^{3}$

Neste artigo, vou sugerir uma interpretação do significado, mas não em termos de referências, nem em termos de usos. Vou argumentar que as experiências de significado dos

\footnotetext{
${ }^{1}$ Para discussões de significado na Educação Matemática, ver, por exemplo, Dobie (2016); Kilpatrick, Hoyles and Skovsmose in collaboration with Valero (Eds.) (2005); Kaiser (2008); Thompson (2013), e Vollstedt (2011).

${ }^{2}$ Ver, por exemplo, Biehler (2005).

${ }^{3}$ Para discussões críticas da modelagem matemática, ver, por exemplo, Jablonka $(2007,2010)$.
} 
estudantes têm a ver com a forma como eles veem suas oportunidades futuras na vida. Eu acho que experiências de significado refletem visualizações de possibilidades. Quando os estudantes não percebem o significado do que estão fazendo na sala de aula de Matemática, pode ser devido ao fato de não poderem conectá-lo ao futuro. Essas ideias fornecem a base para uma interpretação do significado em termos de foregrounds. Com essa interpretação, quero trazer uma outra possibilidade para a discussão do significado na Educação Matemática.

A minha interpretação de significado em termos de foreground não contradiz necessariamente interpretações em termos de referências ou usos. A principal diferença entre as interpretações clássicas e a que eu sugiro diz respeito à dimensão sociopolítica das experiências de significado. Enquanto as interpretações clássicas apresentam um certo grau de indiferença sociopolítica, a interpretação por meio de foreground pode revelar uma formação sociopolítica das experiências de significado dos estudantes.

Por várias vezes escrevi "experiências de significado dos estudantes" e acho importante fazer uma observação sobre essa expressão. Eu a utilizo para destacar que o significado é antes de tudo uma experiência. É possível fazer uma distinção entre significado subjetivo e objetivo e, aqui, eu me concentro no significado subjetivo, que também pode ser denominado significado pessoal (veja Vollstedt, 2011). Isso eu tento destacar usando a palavra "experiência". Em termos gramaticais, a formulação "experiências de significado dos estudantes" poderia indicar que existe um significado (objetivo) do qual os estudantes têm experiência. Mas esta não é a minha interpretação. Eu poderia ter considerado uma terminologia diferente, mas aqui decidi usar a mesma terminologia que usei em publicações anteriores na língua inglesa (students' experiences of meaning).

Na Seção 1, eu apresento um experimento mental que servirá como uma introdução à própria noção de foreground. Na Seção 2, descrevo uma interpretação do significado em termos de foreground. Nas seções 3 a 7, apresento diferentes estruturações sociopolítica de foregrounds e indico o que isso pode significar para as experiências de significado dos estudantes. Na Seção 8, a última, volto à questão de proporcionar significado na Educação Matemática.

\section{Um experimento mental: Paulo e Eloísa}

Vamos considerar dois estudantes, Paulo e Eloísa, ambos nascidos após 2010. Paulo é negro e nasceu numa comunidade carente, da cidade de São Paulo. Sua família é de baixa renda. Eloisa é branca e nasceu num bairro rico de São Paulo. Sua família é da classe média alta. Com 
base nestas informações, as estatísticas indicam algumas possibilidades sobre o futuro destes dois estudantes.

Por exemplo, as estatísticas dizem que Paulo tem a expectativa de viver 69,7 anos, enquanto Eloísa pode esperar viver 80,9 anos; Paulo tem 24,9\% de possibilidades de viver sem água tratada, enquanto Eloísa tem 4,1\%; Paulo tem 7,6\% de chances de vir a ganhar mais que o dobro do salário mínimo, enquanto Eloísa tem 29,9 \%; e a probabilidade de Paulo frequentar o Ensino Superior em universidade é de 3,7\%, enquanto a de Eloísa é 43,0\%. ${ }^{4}$

Como temos que fazer um experimento mental, podemos imaginar muitas coisas: Paulo e Eloísa estudam em escolas distintas e ambas contam com professores que usam uma mesma abordagem pedagógica. Os professores seguem recomendações apresentadas na literatura da Educação Matemática sobre a relevância de tornar os estudantes ativos no seu processo de aprendizagem, em projetos relacionados ao cotidiano. Os professores organizam um mesmo trabalho de projeto: Vamos às férias!Imaginemos, também, que catálogos de agências de viagens foram entregues aos estudantes das duas escolas e eles trabalharam em grupos. Cada grupo foi convidado a escolher o destino para as férias e fazer um orçamento para a viagem, incluindo, também, possíveis despesas de equipamentos extras, como, por exemplo, uma mochila ou uma câmera fotográfica e roupas novas. Assim, os professores seguiram muitas recomendações apresentadas na literatura da Educação Matemática sobre a relevância da contextualização e de como tornar os estudantes ativos no seu processo de aprendizagem, e fizeram o melhor para trazer significado à Educação Matemática.

Poderíamos supor que Paulo e Eloísa experimentariam mais ou menos o mesmo, uma vez que eles estão participando de projetos semelhantes. No entanto, como delineado na estatística, Paulo e Eloísa enfrentam perspectivas de vida radicalmente diferentes. É importante considerar parâmetros sobre expectativa de vida enquanto fazemos interpretações de experiências de significado de Paulo e Eloísa.

Estou propondo uma interpretação de significado com base no conceito de foreground, que ajuda a revelar as complexas formatações sociopolíticas das experiências de significado na Educação Matemática. Eu quero destacar que condições sociais, econômicas, culturais e religiosas, bem como visões, suposições, presunções e preconceitos podem estabelecer algo como significativo ou não para os estudantes.

\footnotetext{
${ }^{4}$ Guilherme Henrique Gomes da Silva me ajudou a organizar esta informação estatística. Ver BRASIL, Ministério do Planejamento (2013) e BRASIL, Instituto Brasileiro de Geografia e Estatística (2014). Veja também Silva (2016).
} 


\section{Foregrounds e significado}

Em várias publicações anteriores, elaborei a noção de foreground. A apresentação principal, no entanto, é encontrada no meu livro Foregrounds (Skovsmose, 2014). Uma linha de inspiração vem de minhas tentativas de interpretar situações na sala de aula de Matemática, em que os estudantes parecem não ter nenhum interesse em aprender. Essas interpretações foram inspiradas pela filosofia, em particular pelas ideias em que a noção de intencionalidade desempenha um papel importante. Essa noção é bem elaborada pela fenomenologia; no entanto minha interpretação de intencionalidade é radicalmente diferente da interpretação na fenomenologia, por isso deixe-me esboçar essa diferença.

A noção de intencionalidade desempenha um papel crucial na forma como Franz Brentano $(1977,1995)$ caracteriza a mente humana, antecipando a formulação da perspectiva fenomenológica de Edmund Husserl. Para Brentano, a intencionalidade é a característica definidora da consciência humana: ela é sempre direcionada para algo. A consciência é um fluxo. Seu direcionamento é um fenômeno a priori; ocorre antes de qualquer fenômeno psicológico particular. Nesse sentido, a intencionalidade será uma categoria humana pura e universal, que precede qualquer forma de estruturação social. Também na formulação de fenomenologia de Husserl (1970, 1998), a intencionalidade funciona como uma categoria básica para interpretar experiências humanas. ${ }^{5}$

Eu também considero a intencionalidade como fundamental para a interpretação das ações humanas. No entanto, discordo totalmente, tanto de Brentano, quanto de Husserl, com respeito à pureza das intencionalidades. Ao contrário, vejo as intencionalidades como estruturadas sociopoliticamente. As direções das intencionalidades são formadas por todos os tipos de fatores sociopolíticos: cultural, religioso e econômico. Elas podem ser direcionadas por presunções, equívocos e qualquer tipo de ideologias. Não há fim para a gama de fatores que podem formar nossas intencionalidades e propósitos para as ações. Nesse sentido, considero as intencionalidades como profundamente estruturadas. Então, as informações fornecidas sobre o futuro de Paulo e Eloísa têm implicações para a formação de intencionalidades deles.

Quando prestamos atenção especial para onde se dirige a intencionalidade, chegamos à noção de foreground. Uma ação pode ser direcionada para possibilidades e o horizonte de possibilidades constitui um foreground. Devemos, no entanto, não apenas referir-nos às possibilidades como sendo fenômenos positivos. Um foreground também é estruturado por

\footnotetext{
${ }^{5}$ Ver Brentano (1977, 1995) e Husserl (1970, 1998, 2001). Veja também Albertazzi (2006) e Smith (1994).
} 
obstáculos e ausência de possibilidades. Algo pode parecer razoável, enquanto outras coisas parecem estar fora de alcance. Neste sentido, os foregrounds são estruturados, tanto em termos de possibilidades como de impossibilidades, por esperanças e aspirações, bem como por medos e aversões.

Considerando a apresentação de Paulo e Eloísa, vemos as profundas diferenças entre seus foregrounds. Como mencionado, essas diferenças são formadas por parâmetros que expressam expectativas de vida, oportunidades de educação, condições econômicas e assim por diante. Elas estabelecem uma profunda estruturação de possibilidades e obstáculos. Um foreground, no entanto, não é uma expressão simples de parâmetros estatísticos. É, também, estruturado através do machismo, do racismo e de presunções em geral. Além disso, um foreground reflete as expectativas, esperanças, medos e frustrações da pessoa. Esta observação não se aplica apenas a Paulo e Eloísa, mas a todos nós. Esta é a ideia básica de se reivindicar uma formação sociopolítica para os foregrounds.

Pode-se falar de foregrounds no plural e essa pluralidade pode ser considerada uma característica intrínseca dos foregrounds. Assim, não faz sentido falar sobre o foreground de uma pessoa como se fosse uma entidade bem definida. A pessoa faz interpretações, muda as interpretações, compreende novas possibilidades ou reconhece novos obstáculos.

A ideia básica de uma interpretação de significado com base no foreground é que as experiências de significado dos estudantes, em primeiro lugar, têm a ver com relações entre as atividades na sala de aula e seus foregrounds. ${ }^{6}$ Aparentemente, as condições de Eloísa diferem das condições de Paulo. Como seus foregrounds são diferentes, suas experiências de significado também podem ser completamente diferentes.

Nas seções seguintes, discuto algumas formações sociopolíticas de foregrounds a fim de indicar como elas estruturam experiências de significado. Falo sobre foregrounds polarizados, foregrounds destruídos, foregrounds amputados, foregrounds direcionadose foregrounds multiplicados.

\section{Foregrounds polarizados}

Em seu estudo Interplay of Citizenship, Education and Mathematics: Formation of Foregrounds of Pakistani Immigrants in Denmark, Sikunder Ali Baber (2007) faz observações

\footnotetext{
${ }^{6}$ Ver também Skovsmose (2016a, 2016b, 2018).
} 
sobre foregrounds de estudantes. ${ }^{7}$

Tais observações são baseadas em minuciosas entrevistas com estudantes e famílias do Paquistão, que vivem na Dinamarca. A princípio, vou me referir a esses estudantes, como estudantes do Paquistão, mesmo que tenham nascido na Dinamarca e falem dinamarquês, sem qualquer sinal de sotaque estrangeiro. Refiro-me aos estudantes com raízes mais longas na Dinamarca como estudantes dinamarqueses. As entrevistas realizadas abordaram as experiências dos estudantes na escola, suas experiências relacionadas com a Matemática e, em particular, como eles veem suas futuras oportunidades na Dinamarca.

Uma das observações importantes de Baber pode ser referida como a polarização dos foregrounds. Os estudantes do Paquistão descreveram suas futuras oportunidades na Dinamarca como sendo diferentes das oportunidades dos estudantes dinamarqueses. Se seus desempenhos na escola são muito acima da média, os estudantes do Paquistão têm as mesmas oportunidades, na Dinamarca, que qualquer estudante dinamarquês. Se, no entanto, seus desempenhos na escola se revelam um pouco acima da média ou na média, sem mencionar se estão abaixo da média, não há outras possibilidades na Dinamarca, a não ser se transformarem em ajudantes no quiosque do pai. Esta expressão pode ser entendida literalmente, ou como uma metáfora para qualquer trabalho não qualificado.

Segundo os estudantes do Paquistão, esta perspectiva polarizada é radicalmente diferente da que se apresenta aos estudantes dinamarqueses. Um estudante dinamarquês médio desfruta de uma gama de oportunidades para o futuro. O foco do estudo de Baber, entretanto, não foi o de verificar até que ponto as oportunidades reais de estudantes do Paquistão estavam polarizadas. Em vez disso, o que quis, de fato, foi identificar a natureza das experiências de estudantes do Paquistão.

A observação de Baber pode estar relacionada a uma outra observação, feita por um dos professores da banca de examinadores de testes nacionais dinamarqueses em Matemática após o último ano na escola, com os estudantes de 15 e 16 anos. Ele me contou sobre um fenômeno observado e que achou estranho. Ele percebeu que diante das perguntas mais difíceis, os estudantes reagiram de duas maneiras diferentes. A maioria dos estudantes deixava o papel em branco. Aparentemente, eles não podiam dar uma resposta e, silenciosamente, reconheceram que não havia mais nada para fazerem. A outra reação, menos comum, foi uma agressão visível quando surgiram questões muito difíceis. As folhas de perguntas foram amassadas; o papel obteve linhas, traços e manchas. O que surpreendeu o professor foi esta última reação agressiva

\footnotetext{
${ }^{7}$ Ver também Baber $(2006,2012)$.
} 
vinda, principalmente, de estudantes imigrantes.

Considerando a observação de Baber, a reação parece menos surpreendente. Ao participar dos testes, alguns dos estudantes imigrantes experimentam que elementos atrativos de seus foregrounds são fundidos em pedaços e substituídos pelo quiosque do pai.

As entrevistas de Baber revelaram que as experiências de significado dos estudantes tinham pouco a ver com a relação entre noções matemáticas e os assuntos que lhes pudessem ser familiares. Ao invés disso, as experiências de significado ficaram sempre relacionadas com possibilidades para o futuro. Em particular, as atividades que tinham função de garantir uma nota melhor foram consideradas significativas.

A polarização de foregrounds é um fenômeno sociopolítico, que pode acontecer para quaisquer grupos de pessoas que vivem em países estrangeiros. Pode se referir a quaisquer grupos de estudantes que foram estigmatizados através de discursos racistas ou presunçosos. Hoje, as hostilidades em relação aos imigrantes na Europa ganham cada vez mais força e, por conseguinte, a polarização dos foregrounds faz parte da realidade política que constitui a experiência de significado para muitos estudantes.

A pesquisa de Barber também revela a maneira profunda como os imigrantes respondem à rotulagem estereotipada, como "Paki"8. Assim, os discursos políticos dinamarqueses agressivos estabelecem olhos suspeitos sobre os imigrantes paquistaneses. Se um único imigrante comete alguma coisa errada, toda a comunidade muçulmana de imigrantes paquistaneses é mapeada. Isso exerce pressão sobre os imigrantes no sentido de se redefinirem. Cria, também, enorme pressão sobre sua formação de foregrounds, que se torna limitada e polarizada. Esta polarização cria a necessidade de vinculação com algum grupo, tais como grupos religiosos em que os imigrantes paquistaneses possam sentir-se confortáveis - embora sem uma escolha religiosa. Desta forma, as experiências de pertencimento, das possibilidades e das impossibilidades, se mostram uma formação sociopolítica.

\section{Foregrounds destruídos}

Em seu estudo, Quem não sonhou em ser um jogador de futebol? Trabalhando com Projetos para Reelaborar Foregrounds, Denival Biotto Filho (2015) apresenta um trabalho de projeto realizado com crianças que vivem em condições sociais vulneráveis.

Como característica inicial, podemos referir-nos ao foreground dessas crianças como

8 "Paki" significa algo negativo ou estereótipos negativos. 
sendo destruídos. Certamente, não faz sentido pensar nisso em termos de autodestruição. Isso tem a ver com as perspectivas de vida que sofreram devido ao contexto no qual estão situadas. Tem a ver com uma destruição que só pode ser contabilizada em categorias sociopolíticas. Ainda assim, são esses foregrounds que promovem experiências de significado ou nãosignificado.

Em que sentido se poderia trabalhar com essas crianças? Não se pode imaginar a mudança dos principais parâmetros sociopolíticos através de iniciativas educativas específicas. O que se poderia esperar, no entanto, é fazer algumas mudanças para alguns estudantes em certas situações. Assim, a educação pode lhes proporcionar novas experiências. O tema do projeto sugerido pelas crianças foi futebol. Claro que houve muitos, muitos projetos sobre futebol antes; no entanto, as crianças estavam ansiosas sobre tal projeto. Assim, as crianças aprenderam sobre a vida de um jogador profissional. Todos nós sabemos sobre a luxuosa vida de jogadores bem conhecidos, retratadas pela mídia. Menos conhecida é a realidade dos profissionais regulares. Um profissional visitou as crianças e, entre muitas informações, ele também explicou que mais da metade dos jogadores de futebol profissionais no Brasil ganham menos do que um salário mínimo.

O projeto de futebol foi concluído com uma apresentação feita pelas crianças para professores, convidados, e alguns pesquisadores da universidade. A apresentação impressionou os adultos. Ali, apareceram as crianças com autoconfiança e entusiasmo. Eles demonstraram profundo conhecimento sobre o tema e o apresentaram com clareza. Depois, um dos professores chorou: nunca imaginara que essas crianças pudessem se apresentar assim.

O projeto de futebol pode ter sido a primeira vez que essas crianças tiveram sucesso em um ambiente educacional. Para uma criança com apenas experiências negativas na escola, as perspectivas de vida se tornam devastadoras, reduzidas; qualquer coisa que pressuponha conhecimento escolar tende a desaparecer de seus foregrounds. No entanto, uma primeira experiência de sucesso na aprendizagem pode estabelecer um novo elemento em um foreground que, de outra forma, foi destruído. Tal elemento de sucesso pode se abrir para novas experiências de significado. Tem a ver com o fenômeno inverso dos estudantes imigrantes sentados no teste nacional experimentando que características relevantes de seus foregrounds se tornam pedaços.

Os foregrounds destruídos são um fenômeno comum. Pode-se pensar nos muitos refugiados que estão à deriva na Europa. Eles deixaram para trás seus pertences, exceto o que foram capazes de levar consigo. O deslocamento causa um dumping sociopolítico e destrói 
foregrounds de muitas pessoas. Quando nos deparamos com estudantes como, por exemplo, Paulo, que, por uma razão ou outra, sofreram dumping sociopolítico, é preciso estarmos muito atentos ao que eles podem experimentar como significativo ou desprovido de significado. A análise dos foregrounds destruídos, bem como as tentativas de fornecer novos elementos aos seus foregrounds, são iniciativas sociopolíticas.

\section{Foregrounds amputados}

Foregrounds podem ser amputados por estereótipos, humilhação e estigmatização de discursos e práticas sociopolíticas. Foregrounds também podem ser amputados devido ao machismo e um exemplo importante tem a ver com mulheres e Matemática.

Durante a década de 1960, o mundo ocidental testemunhou um período de otimismo tecnológico. No entanto, ainda durante este período, percebeu-se uma profunda exclusão de mulheres cursando disciplinas técnicas, incluindo a Matemática. Naquela época, ainda não era fácil para as mulheres caminharem na direção de estudos mais aprofundados da Matemática. As perspectivas dominantes da época excluíam a Matemática dos horizontes femininos.

As amputações dos foregrounds das mulheres devido ao machismo podem eliminar vários elementos de seus foregrounds. Amputações têm várias formas: dependem do período histórico, dos contextos sociopolíticos, das religiões e das culturas. Formas de amputações são expressões de posições sociopolíticas. Tendo os foregrounds drasticamente amputados, podemos imaginar os mesmos destruídos.

Como os demais tipos de foregrounds, aqueles que são amputados também estabelecem condições para experiência de significado (ou não significado). Vamos pensar sobre as prioridades das mulheres na época que estudei na universidade. É possível interpretar as prioridades delas como referências aos seus backgrounds. Assim, podemos nos referir às maneiras como se posicionavam na família e às tradições culturais às quais elas se submetiam. Certamente, é possível obter uma série de interpretações interessantes desta forma. No entanto, quero prestar especial atenção à formação dos foregrounds das mulheres. Esse tipo de interpretação destaca a formação sociopolítica dos foregrounds e proporciona uma compreensão muito mais profunda das experiências de significado e prioridades das mulheres em relação à educação.

Em nossa estatística, comparamos a situação de Paulo e Eloísa. No entanto, também poderíamos ter comparado as estatísticas de Eloísa e de seu irmão Eduardo. Eles vivem nas mesmas condições econômicas; todavia, podem ainda ser diferentes, em relação ao rendimento 
esperado e à educação futura esperada. Essas diferenças podem revelar o nível de machismo que determina suas possibilidades na vida.

Foregrounds podem ser amputados de várias maneiras e por diferentes razões. O machismo causa um formato de amputações. O racismo, outro, sendo importante considera-lo em todas as suas múltiplas formas. Hoje encontramos muitas versões do racismo, por exemplo, como expresso nas hostilidades em relação aos imigrantes. Assim, os estudantes paquistaneses na Dinamarca têm seus foregrounds amputados, como documentado por Baber em termos de foregrounds polarizados.

\section{Foregrounds direcionados}

Quero apresentar uma pesquisa realizada em uma aldeia de indígenas no interior de Estado de São Paulo. ${ }^{9}$ Para esse estudo, o acesso foi autorizado pelo chefe da aldeia e pelo Departamento Federal responsável pela segurança da reserva.

Nesta pesquisa entrevistamos estudantes indígenas. A ideia das entrevistas era conhecer o que os estudantes estavam fazendo nas aulas de Matemática; o que eles tinham de planos e expectativas para o futuro; e como eles viam a relevância da Matemática com respeito a este futuro. Aqui vamos observar os relatos de um dos estudantes, Patrick (pseudônimo)

Tenho 17 anos. Nasci na cidade e vinha para cá de fim de semana. Quando cresci um pouco, resolvi vir morar aqui na aldeia com meu avô. Eu não me acostumava com o jeito da cidade e tudo aqui na aldeia é mais tranquilo. $O$ trabalho é mais puxado, pois a gente tem que ir à roça. A aldeia é bem grande e aqui tem bastante coisa pra fazer. Tem a represa que a gente nada e $o$ campo de futebol. Agora eu não deixo mais a aldeia. A minha namorada tá grávida e nós vamos morar junto. Aqui a gente planta e colhe mandioca. Também tem outras coisa, como a horta. (Skovsmose et al., 2009, p. 246).

Os estudantes foram questionados sobre o que eles gostavam e não gostavam em relação à escola, e esta pergunta foi seguida por outra, sobre o que eles estavam aprendendo em matemática:

Eu fico cansado e tenho que me esforçar muito para prestar mais atenção nos professores. Mas, como eu gosto de estudar, então eu vou.

(Skovsmose et al., 2009, p. 247).

Eu aprendo no cursinho todas as matérias do colegial e até outras. Aprendi os teoremas, equações, raízes, como transformar metros em quilômetros. Eu aprendo um pouco de tudo, pois são as matérias do vestibular. Quando eu era pequeno eu gostava de fazer problemas e de contas de $x$ (multiplicação). Também tem a trigonometria que é difícil e cai muito.

(Skovsmose et al., 2009, p. 249).

\footnotetext{
${ }^{9}$ Ver Skovsmose et al. (2008).
} 
Os estudantes foram então perguntados sobre seus planos para o futuro e sobre o que eles gostariam de fazer:

Eu quero ser enfermeiro e ajudar todos os meus parentes índios a ter mais saúde. Eu queria mesmo trabalhar no postinho de saúde da Funasa. Eu acho até que consigo. Mas, primeiro tenho que estudar para isso. Eu não quero ir morar lá na cidade.

(Skovsmose et al., 2009, p. 252).

Finalmente, foram questionados se viam alguma relevância da Matemática em relação ao trabalho que eles tinham em mente.

Sim. Tudo o que aprendemos na escola, seja de matemática, português ou biologia. Usamos as noções básicas de cada uma. Algumas coisas acho que não tem nada a ver, como as matérias mais dificeis.

(Skovsmose et al., 2009, p. 254).

O significado tem a ver com as esperanças, prioridades e imaginação dos estudantes; tem, também, a ver com superar medos e aversões.

Um foreground fica direcionado quando um elemento específico domina o processo de criação de significado. Este elemento poderia referir-se a objetivos específicos estabelecidos para o futuro, como, por exemplo, poder dominar um determinado tópico matemático. Nesse sentido, o foreground de Patrick ficou direcionado.

Pode-se, também, pensar nos foregrounds dos estudantes imigrantes paquistaneses como direcionados à polarização de suas oportunidades de experiências. Os foregrounds direcionados formam experiências de significado e, certamente, têm a ver com um fenômeno sociopolítico.

\section{Foreground multiplicados}

Na literatura de Educação Matemática crítica e Educação Matemática para justiça social, encontramos muitos exemplos de como abordar importantes questões sociais ${ }^{10}$. A Matemática pode ser usada na leitura e escrita do mundo, como sugerido por Eric Gutstein (2006, 2009, 2012), referindo-se a formulações criadas por Paulo Freire. A leitura do mundo refere-se à maneira como a Matemática pode fornecer ferramentas para interpretar uma gama de fenômenos sociais; e escrever o mundo refere-se à maneira como a Matemática pode ajudar a identificar ações políticas relevantes. Os temas que foram apresentados incluem, por exemplo: poluição, violência, exclusão social, deslocamento, eleição, distribuição de renda, salários

\footnotetext{
${ }^{10}$ Aqui não procuro diferenciar Educação Matemática crítica e Educação Matemática para a justiça social. Veja, por exemplo, Skovsmose (2011).
} 
mínimos, pagamento de impostos, machismo e racismo.

A introdução de tais questões na Educação Matemática representa preocupações sobre como colocar em prática uma Educação Matemática para a justiça social. Assim, parece largamente assumido que os tópicos sociopolíticos são experimentados como significativos pelos estudantes. Sem qualquer hesitação, acho que tais exemplos são relevantes. No entanto, não podemos assumir que as questões políticas e os temas relativos à justiça social, automaticamente, tornem-se significativos pelos estudantes.

Quando os estudantes percebem algo como sendo significativo, isto tem a ver com uma percepção construída. Assim, quando os projetos sobre poluição, violência, exclusão social etc., podem ser vivenciados como significativos pelos estudantes, isso tem muito a ver com os processos interativos complexos que relacionam tais questões aos seus foregrounds.

Este aspecto de uma política de significado pode ser ilustrado pelo estudo de João Luiz Muzinatti (em andamento). Ele está ensinando Matemática para estudantes pertencentes à classe média alta em São Paulo. Sua preocupação é abordar como os argumentos baseados em Matemática podem ajudar a apontar preconceitos amplamente assumidos entre os estudantes com quem ele está trabalhando. Isso poderia, por exemplo, envolver preconceitos com relação aos pobres: eles são preguiçosos; eles não querem trabalhar e ganhar seu próprio dinheiro; e eles custam muito dinheiro à sociedade. João Luiz Muzinatti tenta fornecer uma leitura do mundo por meio da Matemática, que desafia os pressupostos sociopolíticos gerais que fazem parte de uma visão tradicional da classe média. Para mim, este é um exemplo importante de uma política de significado.

O conteúdo do projeto de Muzinatti é semelhante aos projetos propostos, por exemplo, por Eric Gutstein (2006, 2009, 2012) e Marilyn Frankenstein (1983, 1989, 2012), mas em termos de contexto é diferente. Enquanto Gutstein e Frankenstein trabalham com grupos de estudantes que sofrem opressão, Muzinatti investiga grupos em posições econômicas confortáveis. No entanto, ambos os casos estão relacionados a uma política de significado.

Através do seu trabalho, Muzinatti abre brechas no foregrounds de estudantes. Talvez ele abra novas janelas; talvez novos horizontes. De uma maneira mais direta, ele tenta discutir novas preocupações sobre a justiça social. A esse fenômeno eu chamo de multiplicação de foregrounds. Assim, não afirmo que um foreground seja substituído por outro. Em vez disso, vejo que há uma multiplicação, o que significa que os estudantes passam a operar com horizontes diferentes, o que abre diferentes experiências de significado. 


\section{E agora ...}

As observações sobre foregrounds polarizados, destruídos, amputados, direcionados e multiplicados mostram que não existe uma resposta simples para a questão: $O$ que é Educação Matemática significativa? Mas as discussões também mostram que existe muito a fazer para estabelecê-la.

O que fazer? É difícil imaginar mudanças nos principais parâmetros sociopolíticos através de iniciativas educativas específicas na sala de aula. O que se poderia imaginar, no entanto, são algumas mudanças para alguns estudantes, em algumas situações. É possível ficarmos atentos às esperanças dos estudantes; abrir brechas em seus foregrounds; ajudá-los a construir novos elementos em seus foregrounds; e auxiliá-los a multiplicar e reconstruí-los. Dessa maneira, é possível criar novas experiências de significado.

Permitam-me ilustrar isto com a apresentação de duas possibilidades. Primeiro, imaginemos a possibilidade de trabalharmos através das perspectivas apresentadas na introdução nesse artigo. Então, assumimos que os estudantes têm experiências de significado quando trabalham com tópicos familiares. Imaginemos que nós, educadores matemáticos, chegamos ao bairro onde Paulo está vivendo e iniciamos uma cautelosa investigação do que está acontecendo nessa comunidade. Fazemos anotações sobre as condições de se fazer compras, sobre a economia e sobre as coisas que as pessoas tentam fazer para conseguir sobreviver. Com base nisso, criamos um projeto: Nossa Economia. Nós, educadores matemáticos podemos, aqui, identificar todas essas possibilidades para assegurar o significado das atividades matemáticas nas quais vamos engajar os estudantes.

Outra possibilidade é reconhecer que as experiências de significado têm a ver com os foregrounds de estudantes. Vamos imaginar que nós, educadores matemáticos, introduzimos um projeto de Matemática dos Pilotos. Esse projeto poderia se iniciar nessa fina linha de fumaça lá em cima, no alto do céu. Em que direção ela aponta? A que cidade o avião que faz esta linha está se dirigindo? Como usar uma bússola? O que um mapa poderia nos dizer? Se considerarmos o sonho de viajar - o sonho de voar - estaremos abertos à possibilidade de que a Matemática do piloto traga significado à aprendizagem da Matemática, também no bairro de Paulo.

É importante considerar as esperanças que os estudantes têm para o futuro. Temos possibilidades para reconhecer, abrir brechas, reconstruir e ampliar os foregrounds dos estudantes e dessa maneira estabelecer significado em Educação Matemática. 


\section{Agradecimentos}

Este artigo é baseado nos meus artigos "Politics of Meaning in Mathematics Education" (Skovsmose, 2016b) e “Students' Foregrounds and Politics of Meaning in Mathematics Education" (Skovsmose, 2018). Quero agradecer a Ana Carolina Faustino, Amanda Queiroz Moura, João Luiz Muzinatti, Miriam Godoy Penteado, Celia Roncato e Débora Vieira de Souza, pelos seus comentários e sugestões.

\section{Referências}

ALBERTAZZI, L. Immanent realism: an introduction to Brentano. Dordrecht: Springer, 2006.

BABER, S. A. Investigating the Interactions of Foregrounds and Backgrounds of Pakistani Danish Students in Denmark and Learning of Mathematics. In: LAURSEN, E. et al. (Ed.). Tanker til tiden: Om studier af læring. Aalborg: Aalborg Universitet Forlag, 2006. p. 181-201.

BABER, S. A. Interplay of citizenship, education and mathematics: Formation of foregrounds of Pakistani immigrants in Denmark. 2007. 258 f. Tese (Doutorado em Educação Matemática) Department of Learning and Philosophy Aalborg, Aalborg University, 2007.

BABER, S. Learning of Mathematics among Pakistani immigrant children in Barcelona: A sociocultural perspective. In: SKOVSMOSE, O.; GREER, B. (Ed.). Opening the cage: Critique and Politics of Mathematics Education. Rotterdam: Sense Publishers, 2012. p.144-166.

BIEHLER, R. Reconstruction of meaning as a didactical task: The concept of function as an example. In: KILPATRICK, J.; HOYLES, C.; SKOVSMOSE, O. (Ed.). Meaning in mathematics education. New York: Springer, 2005. p. 61-81.

BIOTTO FILHO, D. Quem não sonhou em ser um jogador de futebol? Trabalho com projetos para reelaborar foregrounds. 2015. 234 f. Tese (Doutorado em Educação Matemática) - Universidade Estadual Paulista, Rio Claro, 2015.

BRASIL. Ministério do Planejamento. Orçamento e Gestão. Pesquisa Nacional de Saúde 2013. Brasília: IBGE, 2013.

BRASIL. Instituto Brasileiro de Geografia e Estatística. Síntese de indicadores sociais: uma análise das condições de vida do brasileiro. Brasília: IBGE, 2014.

BRENTANO, F. The psychology of Aristotle: In particular his doctrine of the active intellect. Edição e Tradução de Rolf George. Berkeley: University of California Press, 1977.

BRENTANO, F. Psychology from an empirical standpoint. Tradução de A. C. Rancurello, D. B. Terrell e L. L. McAlister. Introdução de Peter Simons. London: Routledge, 1995.

DOBIE, T. Middle school students' conceptions of the usefulness of mathematics: A sociocultural approach to the study of utility value. Evanston, Illinois: Northwestern University School of Education and Social Policy, 2016. 
FRANKENSTEIN, M. Critical mathematics education: an application of Paulo Freire's epistemology. Journal of Education, Boston, EUA, n. 164, p. 315-339, 1983.

FRANKENSTEIN, M. Relearning mathematics: A different third R - Radical maths. London: Free Association Books, 1989.

FRANKENSTEIN, M. Beyond math content and process: Proposals for underlying aspects of social justice education. In: WAGER, A. A.; STINSON, D. W. (Ed.). Teaching Mathematics for Social Justice: Conversations with Mathematics Educators. USA: NCTM, National Council of Mathematics Teachers, 2012. p. 49-62.

GUTSTEIN, E. Reading and writing the world with mathematics: Toward a pedagogy for social justice. New York and London: Routledge, 2006.

GUTSTEIN, E. Possibilities and challenges in teaching mathematics for social justice. In: ERNEST, P. B.; GREER, B.; SRIRAMAN, B. (Ed.). Critical issues in mathematics education. Charlotte, NC: Information Age Publishing, 2009. p. 351-373.

GUTSTEIN, E. Reflections on teaching and learning mathematics for social justice in urban schools. In: WAGER, A. A.; STINSON, D. W. (Ed.). Teaching mathematics for social justice:

Conversations with mathematics educators. USA: NCTM, National Council of Mathematics Teachers, 2012. p. 63-78.

HUSSERL, E. The crisis of European sciences and transcendental Phenomenology. Tradução e introdução de David Car. Evanston: Northwestern University Press, 1970.

HUSSERL, E. Ideas pertaining to a pure phenomenology and to a phenomenological philosophy: First book. Dordrecht: Kluwer Academic Publishers, 1998.

HUSSERL, E. Logical investigations: Volume 1-2. Tradução de J. N. Findlay. London: Routledge, 2001.

JABLONKA, E. The relevance of modelling and applications: Relevant to whom and for what purpose? In: BLUM, W. et al. (Ed.). Modelling and applications in mathematics education: The 14th ICMI Study. Berlin: Springer, 2007. p. 193-200.

JABLONKA, E. Reflections on mathematical modelling. In: ALRØ, H.; RAVN, O.; VALERO, P. (Ed.). Critical mathematics education: Past, present and future. Rotterdam: Sense Publishers, 2010. p. 89-100.

KAISER, G. Meaning is mathematics education: Reflections from various perspectives, 2008. Disponível em: <https://www.unige.ch/math/EnsMath/Rome2008/WG5/Papers/KAIS.pdf>. Acesso em: 01 de setembro de 2018.

KILPATRICK, J.; HOYLES, C.; SKOVSMOSE, O. (Ed.). Meaning in mathematics education. New York: Springer, 2005.

SILVA, G. H. G. Equidade no acesso e permanência no ensino superior: o papel da educação matemática frente às políticas de ações afirmativas para grupos sub-representados. 2016. $359 \mathrm{f}$. Tese (Doutorado em Educação Matemática) - Universidade Estadual Paulista, Rio Claro, 2016.

MUZINATTI, J. L. (em andamento). A "Verdade" apaziguadora na educação matemática: A argumentação de estudantes e sua visão acerca de injustiça social. Tese (Doutorado em Educação Matemática) - Universidade Estadual Paulista, Rio Claro, em andamento. 
SKOVSMOSE, O. An invitation to critical mathematics education. Rotterdam: Sense Publishers, 2011.

SKOVSMOSE, O. Foregrounds: Opaque stories about learning. Rotterdam: Sense Publishers, 2014.

SKOVSMOSE, O. An intentionality-interpretation of meaning in mathematics education. Educational Studies in Mathematics, Switzerland, v. 90, n. 3, 2016a. p. 411-424.

SKOVSMOSE, O. Politics of meaning in mathematics education. Philosophy of Mathematics Education Journal, n. 31, 2016b. p. 1-15,.

SKOVSMOSE, O. Students' foregrounds and politics of meaning in mathematics education. In: ERNEST, P. (Ed.). The Philosophy of Mathematics Education Today. New York: Springer, 2018. p. 115-130.

SKOVSMOSE, O. et al. "Antes de dividir temos que somar":'entre-vistando' foregrounds de estudantes indígenas. Bolema, Rio Claro, v. 22, n. 34, 2009. p. 237-262.

SMITH, B. Australian philosophy: The legacy of Franz Brentano. Chicago and La Salle: Open Court, 1994.

THOMPSON, P. W. In the absence of meaning... In: LEATHAM, K. (Ed.). Vital directions for research in mathematics education. New York, NY: Springer, 2013. p. 57-93.

VOLLSTEDT, M. Sinnkonstruktion und Mathematiklernen in Deutchland und Hongkong: Eine recokstruktiv-empirische Studie. Wiesbaden: Vieweg + Teuber Verlag, 2011.

WITTGENSTEIN, L. Tractatus logico-philosophicus. London: Routledge \& Kegan Paul, 1922.

WITTGENSTEIN, L. Philosophical investigations. Oxford: Blackwell Publishers, 1997.

Submetido em 19 de Agosto de 2017. Aprovado em 17 de Maio de 2018. 